\title{
Considering fairness in the load shedding scheduling problem
}

\author{
RG Rakotonirainy* \\ I Durbach ${ }^{\dagger}$ \\ J Nyirenda $\ddagger$
}

Received: 15 April 2019; Revised: 2 September 2019; Accepted: 6 September 2019

\begin{abstract}
Every day national power system networks provide thousands of MW of electric power from generating units to consumers, requiring different operations and planning to ensure secure systems. Where demand exceeds supply, load shedding - a controlled, enforced reduction in supply - is necessary to prevent system collapse. Should load shedding need to be implemented, a planned schedule is necessary to allocate geographic areas on the required period of shedding. The problem of how to construct a schedule that fairly allocates load shedding responsibilities over geographic areas with minimum economic impacts is addressed in this paper. Two programming models are proposed. The first model consists of a linear integer programming model in which the objective is to minimise the economic cost subject to different fairness allocation constraints, while the second model involves formulation of the problem as a goal programming model in which different conflicting goals are simultaneously optimised. Several case studies are conducted in the context of a realistic, but hypothetical, scenario to explore the possible solutions that the proposed models provide. Results show that a fair schedule requires a high cost whereas lower cost can only be achieved with some sacrifices to the fairness of the schedule.
\end{abstract}

Key words: Goal programming, Integer programming, Scheduling problem

\section{Introduction}

Electricity infrastructure consists of complex systems of power generation, transmission and distribution $[6,8,15]$. Sufficient quantities of electricity have to be generated from

\footnotetext{
${ }^{*}$ Corresponding author: Department of Statistical Sciences, University of Cape Town, South Africa email: rosephine.rakotonirainy@uct.ac.za

${ }^{\dagger}$ Center for Statistics in Ecology, the Environment, and Conservation, Department of Statistical Sciences, University of Cape Town, South Africa email: indurbach@gmail.com

${ }^{\ddagger}$ Department of Statistical Sciences, University of Cape Town, South Africa email: juwa.nyirenda@uct.ac.za 
generation plants and transmitted through transmission components to meet demand at all times. This system requires a complex mix of management, operations and planning in order to deliver power to consumers. Part of this requirement is the so-called power system operations scheduling [15], which encompasses a set of daily/weekly processes to generate electricity. A diagram illustrating the basic components of these operations is provided in Figure 1.

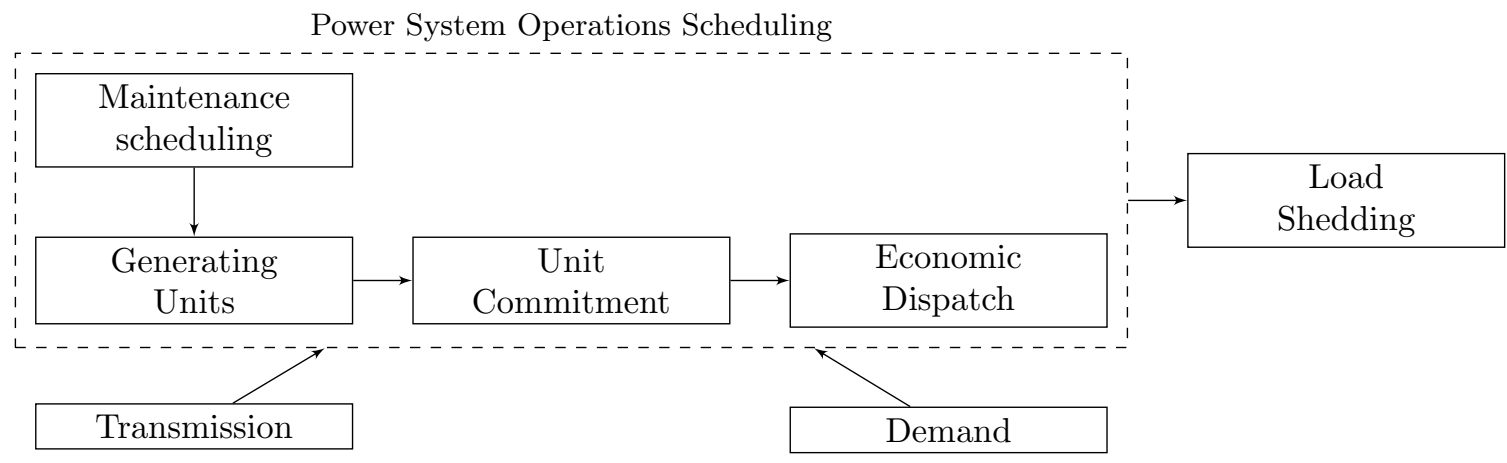

Figure 1: A diagram illustrating the basic operation scheduling in a power system.

Maintenance scheduling entails finding a schedule according to which planned maintenance can be performed on the generating units in a power system. The unit commitment problem, on the other hand, refers to determining which available generating units, those not scheduled for maintenance, should be connected to the power generation system, so as to contribute actively to power generation. Finally, the economic dispatch operation seeks to determine the optimal output from available generating units so as to meet the expected demand at the lowest possible cost $[4,16]$.

Despite these daily maintenances and schedules, shortfalls in forecasted demand or unforeseen failures in generating units may lead to the risk of system collapse. Reserve generation capacity is usually maintained to accommodate such unexpected failures. In the case where this reserve margin is insufficient, load shedding - a planned and controlled reduction in power supply is implemented to compensate the shortage in supply so as to decrease the risk of total system breakdown to acceptable (near-zero) levels.

South Africa has experienced energy shortages since the mid 2000's that have at times been severe, leading to prolonged periods of widespread load shedding. Several causes have been identified. The most important being a rapid increase in electricity demand following the end of the apartheid era in 1994, the lifting of economic sanctions and the provision of a free basic electricity policy in 2001. Construction of power stations and maintenance operations have also at times been delayed, increasing the risk of unit failures and reducing the effectiveness of an already reduced reserve margin [12].

This paper addresses the problem of how to construct a schedule that fairly allocates load shedding responsibilities over geographic areas. Should load shedding need to be implemented, a planned schedule is necessary to allocate areas on the required period of shedding. The ultimate goals of the load shedding scheduling are to fairly allocate areas on the scheduling horizon and also to minimize possible economic impacts. Defining fairness is 
not straightforward and thus various possibilities are considered. The main objective is to emphasize the trade-off between fairness and economic cost. Single and multiple objective integer and goal programming models are proposed to construct optimal schedules. The use of these models are illustrated in the context of a hypothetical, but realistic, test case of determining load shedding schedules for the City of Cape Town.

The remainder of the paper is organised as follows. In $\S 2$, a brief background on the problem, as well as existing literature study concerning the same, are presented. This is followed by a description of the proposed models in $\S 3$. Details of the hypothetical data employed and the experimental studies conducted are presented in $\S 4$. The computational results are reported and discussed in $\S 5$. Some final remarks follow in $\S 6$.

\section{The problem in context}

Eskom, the parastatal producer and distributor of electricity in South Africa, is responsible for $95 \%$ of South Africa's power generation, and is also responsible for the implementation of load shedding. Load shedding protocols dictate that the power system be balanced at $50 \mathrm{~Hz}$, and that when the national electricity grid is under pressure the load on the grid be reduced to restore this balance. Load reduction consists of a two-phase process. First, large industrial customers can be instructed, by prior agreement, to reduce their consumption by up to $20 \%$, a process known as load curtailment. If demand still exceeds supply after load curtailment, or there is insufficient time to implement curtailment, a second phase — load shedding — is implemented [3].

Eskom implements load shedding by first determining the necessary reduction in load, depending on the generation capacity shortfall at a particular time. These are implemented as three discrete "stages", with stage 1 reflecting a generation shortfall of up to $1000 \mathrm{MW}$, stage 2 a shortfall of up to $2000 \mathrm{MW}$, and stage 3 a shortfall of up to $4000 \mathrm{MW}$. Depending on the load shedding stage, parts of the network are switched off according to a planned, rotational schedule that varies by day and time of day. These schedules are published in advance and are publicly available. An example of a daily schedule for the City of Cape Town is shown in Table 1.

\begin{tabular}{|c|c|c|c|c|c|c|c|c|c|c|c|c|}
\hline & $\begin{array}{c}00: 00 \\
\text { to } \\
02: 30\end{array}$ & $\begin{array}{c}02: 00 \\
\text { to } \\
04: 30\end{array}$ & $\begin{array}{c}04: 00 \\
\text { to } \\
06: 30\end{array}$ & $\begin{array}{c}06: 00 \\
\text { to } \\
08: 30\end{array}$ & $\begin{array}{c}08: 00 \\
\text { to } \\
10: 30\end{array}$ & $\begin{array}{c}10: 00 \\
\text { to } \\
12: 30\end{array}$ & $\begin{array}{c}12: 00 \\
\text { to } \\
14: 30\end{array}$ & $\begin{array}{c}14: 00 \\
\text { to } \\
16: 30\end{array}$ & $\begin{array}{c}16: 00 \\
\text { to } \\
18: 30\end{array}$ & $\begin{array}{c}18: 00 \\
\text { to } \\
20: 30\end{array}$ & $\begin{array}{c}20: 00 \\
\text { to } \\
22: 30\end{array}$ & $\begin{array}{c}22: 00 \\
\text { to } \\
00: 30\end{array}$ \\
\hline $\begin{array}{c}\text { Stage } \\
1\end{array}$ & 5 & 6 & 7 & 8 & 9 & 10 & 11 & 12 & 13 & 14 & 15 & 16 \\
\hline $\begin{array}{c}\text { Stage } \\
2\end{array}$ & 13,5 & 14,6 & 15,7 & 16,8 & 1,9 & 2,10 & 3,11 & 4,12 & 5,13 & 6,14 & 7,15 & 8,16 \\
\hline $\begin{array}{c}\text { Stage } \\
3 \mathrm{~A}^{*}\end{array}$ & $\begin{array}{c}5,13 \\
1\end{array}$ & $\begin{array}{c}6,14 \\
2\end{array}$ & $\begin{array}{c}7,15 \\
3\end{array}$ & $\begin{array}{c}8,16 \\
4\end{array}$ & $9,1,5$ & $\begin{array}{c}10,2, \\
6\end{array}$ & $\begin{array}{c}11,3 \\
7\end{array}$ & $\begin{array}{c}12,4, \\
8\end{array}$ & $\begin{array}{c}13,5, \\
9\end{array}$ & $\begin{array}{c}14,6 \\
10\end{array}$ & $\begin{array}{c}15,7 \\
11\end{array}$ & $\begin{array}{c}16,8, \\
12\end{array}$ \\
\hline $\begin{array}{c}\text { Stage } \\
3 B^{*}\end{array}$ & $\begin{array}{l}1,9 \\
13,5\end{array}$ & $\begin{array}{c}2,10 \\
14,6\end{array}$ & $\begin{array}{c}3,11 \\
15,7\end{array}$ & $\begin{array}{c}4,12 \\
16,8\end{array}$ & $\begin{array}{c}5,13 \\
1,9\end{array}$ & $\begin{array}{c}6,14 \\
2,10\end{array}$ & $\begin{array}{l}7,15 \\
3,11\end{array}$ & $\begin{array}{l}8,16 \\
4,12\end{array}$ & $\begin{array}{l}9,1 \\
5,13\end{array}$ & $\begin{array}{c}10,2 \\
6,14\end{array}$ & $\begin{array}{l}11,3 \\
7,15\end{array}$ & $\begin{array}{l}12,4, \\
8,16\end{array}$ \\
\hline
\end{tabular}

Table 1: An example of a daily load shedding schedule for the City of Cape Town. Entries in the table indicate areas in the City. 
Load shedding is often, but not always, predicted in advance, allowing consumers a measure of control over how they organise their days/weeks to minimize economic costs and general inconvenience. Nevertheless, it has been reported that load shedding has had a serious and negative impacts on economic and social life of the South Africa population since its start [5].

Tackling the symptoms of pervasive, systematic load shedding as seen in South Africa is fundamentally different to how load shedding is often treated elsewhere - as an engineering problem involving an automated correction of short-term instability in the system. In the literature, load shedding is incorporated in the form of reliability constraints in the unit commitment problem, referred to as the reliability-constrained unit commitment problem [11,13]. The objective of reliability constraints is to ensure that sufficient reserve is maintained so that the probability of having power deficiency in the schedule is lower than a pre-specified threshold, i.e. the maximum probability of loss of load [17].

While technical constraints remain critical, other objectives must also play a role in strategic decisions around load shedding. Broadly speaking, the current paper aims to construct such tools for supporting strategic decision making around load shedding. The focus is specifically to develop programming models which may be employed to generate feasible and fair schedules should load shedding need to be implemented.

\section{$3 \quad$ Model formulations and approaches}

Several studies related to scheduling problems may be found in the literature $[2,9,10,14]$. Among the most studied problems is the job scheduling problem, which consists of finding an optimal schedule that specifies when and on which machine certain jobs are to be executed in order to minimise the average completion time as well as its makespan [10]. Another well-studied scheduling problem is the staff scheduling problem, which consists of assigning employees to working shifts over a given period of time such that personnel policies or individual preferences are satisfied while minimising the total cost [14].

In the load shedding context, the scheduling process consists of allocating a set of shedable areas over time and day slots according to the given amount of load to shed and subject to several constraints. The objectives are to minimise the economic cost and also to maximise the allocation fairness. This section contains a detailed description of the problem formulation, as well as the two proposed models.

\subsection{Model variables}

Suppose there are $r$ areas to be shed in the city, indexed by the set $\mathcal{R}=\{1, \ldots, r\}, t$ decision time slots over the day, indexed by the set $\mathcal{J}=\{1, \ldots, t\}$, and $p$ decision day slots over the scheduling horizon, indexed by the set $\mathcal{K}=\{1, \ldots, p\}$. Define the binary decision variable $x_{i j k}$ to take the value one if area $i \in \mathcal{R}$ is assigned to be shed on day $k \in \mathcal{K}$ at a specific time $j \in \mathcal{J}$, or zero otherwise. Let $c_{i j k}$ be the estimated cost associated with an area $i \in \mathcal{R}$ if it is shed during a time period $j k$ with $j \in \mathcal{J}$ and $k \in \mathcal{K}$. 


\subsection{Models objectives}

The first model objective adopted in this paper is to minimise the total economic cost, sum of $c_{i j k} x_{i j k}$, associated with each shed area $i \in \mathcal{R}$ over time periods $j \in \mathcal{J}$ and $k \in \mathcal{K}$, that is

$$
\operatorname{minimise} \sum_{i \in \mathcal{R}} \sum_{j \in \mathcal{J}} \sum_{k \in \mathcal{K}} c_{i j k} x_{i j k} \text {. }
$$

The second model objective consists of maximising the fairness allocation. Two types of fairness allocations are considered in the models: Rotational fairness and Cumulative fairness. The objective in the rotational fairness is to avoid successive times shed of a given area during the scheduling horizon, while the cumulative fairness ensures that the average number of times each area is shed during the scheduling horizon is balanced. An example of a schedule which satisfies both rotational and cumulative fairness objectives is provided in Table 2. In this example, all areas have been shed with the same amount of times (6 times), and no repetition in the shedding time or day slot of each area is perceived.

\begin{tabular}{|c|c|c|c|c|c|c|}
\hline Period & Monday & Tuesday & Wednesday & Thursday & Friday & Saturday \\
\hline 07:00 - 09:00 & Area 1 & Area 2 & Area 3 & Area 4 & Area 5 & Area 6 \\
\hline 09:00 - 11:00 & Area 2 & Area 3 & Area 4 & Area 5 & Area 6 & Area 1 \\
\hline $11: 00-13: 00$ & Area 3 & Area 4 & Area 5 & Area 6 & Area 1 & Area 2 \\
\hline $13: 00-15: 00$ & Area 4 & Area 5 & Area 6 & Area 1 & Area 2 & Area 3 \\
\hline $15: 00-17: 00$ & Area 5 & Area 6 & Area 1 & Area 2 & Area 3 & Area 4 \\
\hline $17: 00-19: 00$ & Area 6 & Area 1 & Area 2 & Area 3 & Area 4 & Area 5 \\
\hline
\end{tabular}

Table 2: An example of a schedule which satisfies both rotational and cumulative fairness objectives.

The use of these objectives, and how they are measured quantitatively, was validated during an informal workshop with eight energy experts working at the Energy Research Center at the University of Cape Town. The workshop took the form of a structured discussion of the load shedding scheduling problem. It began with a brief presentation of the load shedding scheduling problem, including some preliminary models that illustrates how the economic costs and fairness allocations objectives are implemented. A worked example was shown to the group to demonstrate the working of the models. The group was asked to evaluate and discuss what objectives are important in the scheduling problem. The group agreed that economic cost and fairness were important. Some members have suggested that predictability is also an important objective that could be included in the model. A schedule is predictable if for a given period of shedding time, areas that should be shed in these slots are known in advance. The focus in this study is, however, to develop models that are able to spontaneously generate schedule should load shedding need to be implemented. Potential areas to be shed are not known in advance, therefore this objective was not included in the model. 
The group was also asked whether the notion of rotational fairness (per day and per time slot), and cumulative fairness adequately captured the main aspects of fairness. The group agreed that these were suitable attributes, and did not add any further suggestions on this topic.

\subsection{The first model: Single objective allocation approach}

The first scheduling model proposed in this paper is primarily an integer programming based approach whose decision variables are the planned scheduling period of the different areas. The first objective, economic cost, described in $\S 3.2$ is the only objective to be minimised in this case, while the fairness allocation objectives are formulated as constraints of the problem.

Let $d_{i j k}$ be the load demand forecast of an area $i \in \mathcal{R}$ on day $k \in \mathcal{K}$ at a specific time $j \in \mathcal{J}$, and $l_{j k}$ be the required amount of load to be shed with respect to a specific time period $j k$, with $j \in \mathcal{J}$ and $k \in \mathcal{K}$. Let $\lambda$ be an integer, which can take on values that lie in the set $\left\{1, \ldots,\left\lfloor\frac{s t p}{r}\right\rfloor\right\}^{1}$, where $s$ represents the load shedding stage or the minimum required number of areas to be shed during each time period ${ }^{2}$, and $\alpha$ and $\beta$ two parameters which can take on values ranging in $\{1, \ldots, p\}$ and $\{1, \ldots, t\}$, respectively.

The objective in the first load shedding scheduling model is to

$$
\begin{aligned}
\text { minimise } & \sum_{i \in \mathcal{R}} \sum_{j \in \mathcal{J}} \sum_{k \in \mathcal{K}} c_{i j k} x_{i j k} \\
\text { subject to } & \sum_{i \in \mathcal{R}} d_{i j k} x_{i j k} \geq l_{j k}, \quad j \in \mathcal{J}, k \in \mathcal{K}, \\
& \sum_{j \in \mathcal{J}} \sum_{k \in \mathcal{K}} x_{i j k} \geq \lambda, \quad i \in \mathcal{R}, \\
& \sum_{k \in \mathcal{K}} x_{i j k} \leq \alpha\left\lceil\frac{s p}{r}\right\rceil, \quad i \in \mathcal{R}, j \in \mathcal{J}, \\
& \sum_{j \in \mathcal{J}} x_{i j k} \leq \beta\left\lceil\frac{s t}{r}\right\rceil, \quad i \in \mathcal{R}, k \in \mathcal{K}, \\
& x_{i j k} \in\{0,1\}, \quad i \in \mathcal{R}, j \in \mathcal{J}, k \in \mathcal{K} .
\end{aligned}
$$

The objective function in (1) represents the total economic cost. Constraint set (2) ensures that the required amount of load to be shed during each time slice is satisfied. The cumulative fairness is ensured by constraint (3). The parameter $\lambda$ in this constraint determines the level of fairness with respect to the total number of times each area is shed. That is, it ensures that all areas are shed with the same number of time periods on average. A value of $\lambda$ equal to one, for example, indicates that all areas should at least be shed once during the schedule horizon.

\footnotetext{
${ }^{1}\lfloor x\rfloor$ is the floor integer part of the number $x$, while $\lceil x\rceil$ denotes its ceiling integer part.

${ }^{2}$ According to the implemented schedule for the City of Cape Town, see Figure 2 for example, the values of $s$ range from $\{1, \ldots, 4\}$.
} 
The rotational fairness involves both daily and hourly rotation allocations, respectively, represented by constraints (4) and (5). The daily rotation allocation constraint ensures that no area should be scheduled for shedding on the same time slot over the scheduling horizon until all other areas have been shed in that particular slot. Analogously, the hourly allocation constraint ensures that no repetition should be allowed with respect to the period of a day an area has been allocated to, that is an area should not be scheduled for shedding on the same day until the remaining areas have been shed on that day. The level of fairness with respect to these two constraints is adjusted with the two parameters $\alpha$ and $\beta$. A value of $\alpha$ (resp. $\beta$ ) equal to three, for example, indicates that an area can be shed three times at the same period slot over the scheduling horizon (resp. an area can be shed three times at different time slot on the same day).

\subsection{The second model: Multiple objective allocation approach}

The second scheduling model consists of a multi-objective programming model in which the two objectives stated in 3.2 are simultaneously optimised. More precisely, the objective function (1) together with the set of constraints (3)-(5) in the first model of $\S 3.3$ are converted into a set of goals that need to be achieved simultaneously. Each goal is associated with a target value and the objective in this case consists of minimising the weighted sum of deviations of all goals to their corresponding targets. This approach provides a more flexible way to model the problem, as decisions makers can customize the targets' values according to their preferences.

The first goal consists of minimising the total economic cost

$$
\sum_{i \in \mathcal{R}} \sum_{j \in \mathcal{J}} \sum_{k \in \mathcal{K}} c_{i j k} x_{i j k}
$$

its target is denoted by $E$. The second goal is the cumulative fairness determined by the set

$$
\left\{\sum_{j \in \mathcal{J}} \sum_{k \in \mathcal{K}} x_{i j k}, i \in \mathcal{R}\right\}
$$

whose target is denoted by

$$
\left\{F_{i}^{\text {overall }}, i \in \mathcal{R}\right\}
$$

The last two goals are related to the daily and hourly rotational allocations,

$$
\left\{\sum_{k \in \mathcal{K}} x_{i j k}, i \in \mathcal{R}, j \in \mathcal{J}\right\}
$$

and

$$
\left\{\sum_{j \in \mathcal{J}} x_{i j k}, i \in \mathcal{R}, k \in \mathcal{K}\right\}
$$


whose respective targets are represented by

$$
\left\{F_{i j}^{(\text {day })}, i \in \mathcal{R}, j \in \mathcal{J}\right\}
$$

and

$$
\left\{F_{i k}^{(\text {time })}, i \in \mathcal{R}, k \in \mathcal{K}\right\}
$$

It is assumed that goals which belong to the same set have similar target values. Define the binary variable $z$ to take value of one if there are more time slots available than areas, that is if $t p>r$, or zero otherwise.

The objective in the second model is then to

$$
\begin{aligned}
& \operatorname{minimise} \omega d+\sum_{i \in \mathcal{R}} \omega_{i}^{\text {(overall) }} d_{i}^{\text {(overall) }}+\sum_{i \in \mathcal{R}} \sum_{k \in \mathcal{K}} \omega_{i k}^{\text {(time) }} d_{i k}^{\text {(time) }}+\sum_{i \in \mathcal{R}} \sum_{j \in \mathcal{J}} \omega_{i j}^{\text {(day) }} d_{i j}^{\text {(day) }} \\
& \text { subject to } \sum_{i \in \mathcal{R}} d_{i, j, k} x_{i j k} \geq l_{j k}, \quad j \in \mathcal{J}, k \in \mathcal{K} \text {, } \\
& \sum_{j \in \mathcal{J}} \sum_{k \in \mathcal{K}} x_{i j k} \geq z, \quad i \in \mathcal{R}, \\
& \sum_{i \in \mathcal{R}} \sum_{j \in \mathcal{J}} \sum_{k \in \mathcal{K}} c_{i j k} x_{i j k}-d \leq E, \\
& \sum_{j \in \mathcal{J}} \sum_{k \in \mathcal{K}} x_{i j k}-d_{i}^{\text {(overall) }} \leq F_{i}^{\text {(overall) }}, \quad i \in \mathcal{R}, \\
& \sum_{k \in \mathcal{K}} x_{i j k}-d_{i j}^{\text {(day) }} \leq F_{i j}^{\text {(day) }}, \quad i \in \mathcal{R}, j \in \mathcal{J}, \\
& \sum_{j \in \mathcal{J}} x_{i j k}-d_{i k}^{(\text {time })} \leq F_{i k}^{(\text {time })}, \quad i \in \mathcal{R}, k \in \mathcal{K}, \\
& x_{i j k} \text { binary, } i \in \mathcal{R}, j \in \mathcal{J}, k \in \mathcal{K}, \\
& d, d_{i}^{\text {(overall) }}, d_{i j}^{\text {(day) }}, d_{i k}^{\text {(time) }} \geq 0 \text {. }
\end{aligned}
$$

The objective function in (7) represents the weighted sum deviations of all goals to their corresponding targets, where $\omega$ is the weight associated with the economic cost goal,

$$
\omega_{i}^{(\text {overall })}(i \in \mathcal{R})
$$

is the weight associated with the cumulative fairness goal, and

$$
\omega_{i j}^{\text {(day) }}(i \in \mathcal{R}, j \in \mathcal{J})
$$


and

$$
\omega_{i k}^{\text {(time) }}(i \in \mathcal{R}, k \in \mathcal{K})
$$

are the weights associated with the daily and hourly rotational allocations goals, respectively. The variable $d$ represents the deviation of the economic cost goal from its target value as illustrated in (10). Analogously, the deviation variable

$$
d_{i}^{(\text {overall })}(i \in \mathcal{R})
$$

denotes the difference between the cumulative fairness goal to its target

$$
F_{i}^{(\text {overall })}(i \in \mathcal{R})
$$

(as shown in constraint (11)), and

$$
d_{i j}^{\text {(day) }}(i \in \mathcal{R}, j \in \mathcal{J}), d_{i k}^{(\text {time })}(i \in \mathcal{R}, k \in \mathcal{K})
$$

express the deviations of the daily and hourly rotational allocations goals from their target values

$$
F_{i j}^{(\text {day })}(i \in \mathcal{R}, j \in \mathcal{J}), F_{i k}^{\text {(time) }}(i \in \mathcal{R}, k \in \mathcal{K})
$$

respectively (as presented in constraints (12)-(13)). Constraint set (8) ensures that the required amount of load to be shed during each time window is satisfied. Finally, constraint set (9) ensures that each area is at least assigned once for shedding over the scheduling horizon in case there are more time slots available than areas.

\section{Hypothetical data and experimental study}

In order to illustrate the working of the single- and multi-objective allocation models described in $\S 3.3$ and $\S 3.4$, a hypothetical data set was generated. The data entail electricity consumption and estimated economic costs associated with load shedding for all areas in the City of Cape Town. The two models are solved in the context of this hypothetical data. A limited experimental study based on sensitivity analysis with respect to the underlying parameters of the models is also performed in order to assess their performances. Details of the hypothetical data, as well as the computational studies conducted, are provided in this section. It is noteworthy that the data considered in this study are hypothetical data and for a real implementation one would definitely want to assess the real values. 


\subsection{Hypothetical data}

Planners for the City of Cape Town divide the City into $r=16$ areas, and use a daily or monthly scheduling horizon [7]. For simplicity purposes, the scheduling horizon is defined by $t=12$ hours and $p=7$ days in this study. Data on the proportion of mixed use, industrial and commercial sectors for each area was provided by the City of Cape Town. Moreover, data about the population and number of households in each area is obtained from [1]. Data with respect to the economic costs caused by load shedding for all areas are not available; estimated costs were thus employed in the current study. These costs were calculated such that the economic costs associated with the different sectors within an area are taken into consideration. More specifically, for each area, an estimated cost is given to each of the economic sectors within the area and the sum of these costs represents the total economic cost associated to that specific area.

For each area, denote by $c_{M}(j k)$ the cost of load shedding associated with its corresponding mixed use sector in Rand per $\mathrm{km}^{2}, c_{I}(j k)$ the economic cost for its industrial sector in Rand per $\mathrm{km}^{2}, c_{C}(j k)$ the economic cost for its commercial sector in Rand per $\mathrm{km}^{2}$, and $c_{H}(j k)$ the economic cost associated with its households in Rand per household, all at specific time period $j k, j \in\{1, \ldots, 12\}$ and $k \in\{1, \ldots, 7\}$. It is assumed that all these costs are the same for all areas and their values are presented in Table 3. These values were selected in such a way that possible significant impact and peak hours were taken into account.

\begin{tabular}{|c|c|c|c|c|}
\hline Time period & $\begin{array}{c}\text { Mixed use } \\
c_{M}\left(R / \mathbf{k m}^{2}\right)\end{array}$ & $\begin{array}{c}\text { Industrial } \\
c_{I}\left(R / \mathbf{k m}^{2}\right)\end{array}$ & $\begin{array}{l}\text { Commercial } \\
c_{C}\left(R / \mathrm{km}^{2}\right)\end{array}$ & $\begin{array}{c}\text { Households } \\
c_{H}(\mathrm{R} / \text { household })\end{array}$ \\
\hline 00:00 - 02:00 & 600 & 550 & 550 & 5 \\
\hline $02: 00-04: 00$ & 600 & 550 & 550 & 5 \\
\hline 04:00 - 06:00 & 600 & 580 & 550 & 5 \\
\hline 06:00 - 08:00 & 620 & 600 & 560 & 1 \\
\hline 08:00 - 10:00 & 750 & 700 & 650 & 1 \\
\hline $10: 00-12: 00$ & 800 & 750 & 700 & 1 \\
\hline $12: 00-14: 00$ & 790 & 750 & 700 & 1 \\
\hline $14: 00-16: 00$ & 810 & 760 & 700 & 1 \\
\hline $16: 00-18: 00$ & 740 & 700 & 700 & 5 \\
\hline $18: 00-20: 00$ & 700 & 660 & 650 & 5 \\
\hline $20: 00-22: 00$ & 650 & 620 & 620 & 5 \\
\hline $22: 00-00: 00$ & 610 & 600 & 580 & 5 \\
\hline
\end{tabular}

Table 3: Estimated economic costs caused by load shedding associated with all sectors and households in all areas.

Measuring and interpreting the costs associated with households are more difficult than in the case of industrial, commercial, or mixed use sectors. Households costs can be partly interpreted as direct monetary costs, because load shedding means that substitutes must be found for electricity-consuming goods and services, and these will often be more expensive (e.g. boiling water on a gas stove). However, load shedding also causes an inconvenience to households that is not directly measurable in monetary terms. In principle, one could estimate a "willingness to pay" (to avoid load shedding) associated with households, either in aggregate or individually manner. In this study, the approach is rather to set household costs in each time period so that total household costs are roughly of the same magnitude 
as the sum of costs across other sectors (commercial, industrial, mixed). That is, the household costs are selected so that these costs neither swamp, nor are swamped by, the costs of the other sectors.

The surface area occupied by each sector within each area was calculated using the data from the City of Cape Town. Denote by $s_{M}(i)\left(\right.$ in $\left.\mathrm{km}^{2}\right), s_{I}(i)\left(\right.$ in $\left.\mathrm{km}^{2}\right)$ and $s_{C}(i)\left(\right.$ in $\left.\mathrm{km}^{2}\right)$ the corresponding surfaces with respect to mixed use, industrial and commercial sectors, respectively. The estimated cost associated with an area $i, i \in\{1, \ldots, 16\}$ at a specific time period $j k, j \in\{1, \ldots, 12\}$ and $k \in\{1, \ldots, 7\}$ is, therefore, determined by

$$
c_{i j k}=s_{M}(i) c_{M}(j k)+s_{I}(i) c_{I}(j k)+s_{C}(i) c_{C}(j k)+\operatorname{households}(i) c_{H}(j k) .
$$

Regional electricity consumption data is also not available, thus a hypothetical case scenario was developed. It was assumed that all areas have different electricity demand which can be less or greater than the required amount of load to be shed with respect to each time period, and that the difference between the maximum and minimum demand across all areas is relatively large. That is, areas which predominantly populated by industrial and commercial sectors were assigned with higher electricity demand than the other areas, as they were expected to consume more electricity as compared to the remaining areas. To do so, the 16 areas were ranked based on the total surfaces occupied by the various sectors within the areas and each area was associated a load demand that is proportional to its rank. The expected demand of an area $i, i \in\{1, \ldots, 16\}$ with a rank $u, u \in\{1, \ldots, 16\}$ is therefore determined by

$$
D_{i}(u)=\frac{0.9^{u}}{\sum_{r} 0.9^{u}} .
$$

\subsection{Experimental study}

A limited computational study based on experimental design was carried out according to which suitable parameter values may be selected for the two models of $\S 3.3$ and $\S 3.4$. The experimental design consisted of testing various parameter settings of the two models. These parameters are the level of fairness, $\lambda, \alpha$, and $\beta$, with respect to the cumulative and rotation allocations constraints for the single-objective model, and the weights, $\omega, \omega_{i}^{\text {(overall) }}, \omega_{i k}^{\text {(time) }}$, and $\omega_{i j}^{\text {(day) }}$, associated with all goals for the multi-objective model.

Five values, in the range $\{1, \ldots, 5\}$, were considered for each of the parameters $\lambda$, $\alpha$, and $\beta$. These values were chosen for simplicity purposes, but each parameter can be varied between their minimum and maximum values. Moreover, $\alpha$ and $\beta$ were assumed to have identical values for illustration purposes.

With regard to the weights associated to the various goals in the multi-objective model, two test cases were implemented. In the first case, the weight associated to the economic cost goal was varied, with an increasing magnitude from 1 to 7 , while the other weights were kept constant. In the second case, the weights $\omega_{i k}^{\text {(time) }}$ and $\omega_{i j}^{\text {(day) }}$ associated to the daily and hourly rotational allocations goals were varied, with an increasing magnitude from 1 to 7, while keeping the remaining weights constant. 
Note that the different goals considered in the multiple objective formulation are measured on different scales. In order to adjust those scales, so as to obtain a dimensionless objective function, the weight associated to each goal was set to be equal to the inverse of the range of value of its corresponding goal, which may be calculated as the difference between the maximum and minimum value of the target obtained from the single objective allocation model. Solutions obtained during a preliminary computational run of the single objective model suggested the following range of values, $R V$, for each weight:

$$
\begin{aligned}
R V(\omega) & =181356 \\
R V\left(\omega_{i}^{\text {(overall })}\right) & =30, \quad i \in\{1, \ldots, 16\}, \\
R V\left(\omega_{i j}^{\text {(day })}\right) & =5, \quad i \in\{1, \ldots, 16\}, j \in\{1, \ldots, 12\}, \\
R V\left(\omega_{i k}^{\text {(time })}\right) & =5, \quad i \in\{1, \ldots, 16\}, k \in\{1, \ldots, 7\} .
\end{aligned}
$$

Moreover, for illustration purposes, the values of the various targets in the multiple objective formulation are fixed, with

$$
\begin{aligned}
E & =250000, \\
F_{i}^{\text {(overall) }} & =\left\lfloor\frac{s t p}{r}\right\rfloor, \quad i \in\{1, \ldots, 16\}, \\
F_{i j}^{\text {(day) }} & =\left\lceil\frac{s p}{r}\right\rceil, \quad i \in\{1, \ldots, 16\}, j \in\{1, \ldots, 12\}, \\
F_{i k}^{\text {(time) }} & =\left\lceil\frac{s t}{r}\right\rceil, \quad i \in\{1, \ldots, 16\}, k \in\{1, \ldots, 7\} .
\end{aligned}
$$

The values of all fairness goals targets were set to be the (near-)optimal values that could be obtained with a fairly scheduling plan (i.e. without considering any possible economic costs). The value of the economic cost goal target was chosen so that it is moderate, not excessively demanding. These values can, however, be customized according to the decision maker preferences.

\section{Results and discussion}

The numerical results obtained when following the experimental design described in $\S 4.2$ are presented in this section. The results returned by the single objective allocation model are first reported, which is followed by a discussion of the results returned by the multiple objective model. The models were run and solved in Microsoft Excel, employing the Excel Solver package.

\subsection{Results obtained by the single objective model}

An area chart of the solutions returned by the single objective allocation model, when implemented with different values of $\lambda, \alpha$, and $\beta$, is shown in Figure 2. It illustrates the 
impact of varying the values of the aforementioned three parameters on the total economic costs obtained from solving the model.

It is clear from the figure that a large value of $\lambda(\lambda \geq 3)$ results in high economic costs, while for a small value of $\lambda(\lambda<3)$ the total economic costs are relatively low. This is explained by the fact that the parameter $\lambda$ adjusts the level of fairness with respect to the total number of times each area is shed during the scheduling horizon. A small value of $\lambda$, for example $\lambda=1$, suggests that all areas should at least be shed at least once during the planning horizon, hence allowing areas with low economic impact to be shed more often compared to other areas. In contrast, a large value of $\lambda$ indicates that all areas are shed almost an equal number of times, regardless of their associated economic costs.

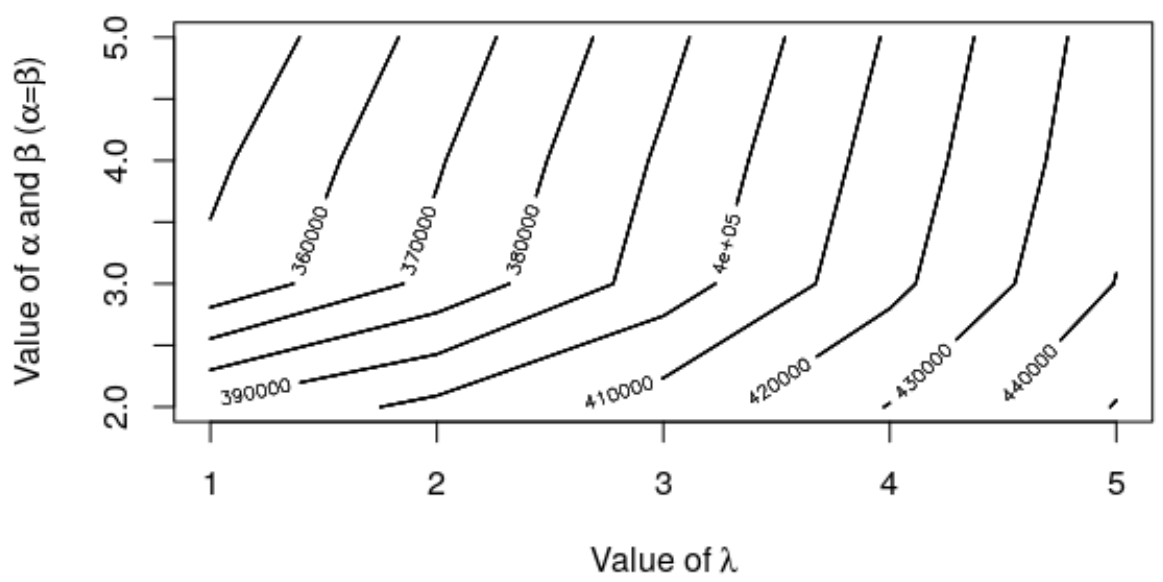

Figure 2: $\quad$ The effect of varying the values of the three parameters $\lambda, \alpha$ and $\beta$ on the total economic cost of a solution obtained by solving the single objective model.

Furthermore, a reduction on the total economic cost can be obtained with large values of the two parameters $\alpha$ and $\beta$. A value of these two parameters equal to 5 , for example, suggests that an area can be shed five times a day and five times in the same time period over the schedule horizon, thus a possible schedule solution is to repeatedly shed areas with low economic costs on any peak time periods.

Histograms of the distribution of the number of times shed of all areas when solving the single objective model for different values of the parameters $\lambda, \alpha$, and $\beta$ are given in Figure 3. For $\lambda=5, \alpha=\beta=5,75 \%$ of the total number of areas are shed five times in the returned schedule. On the other hand, for $\lambda=1$ and $\alpha=\beta=5,25 \%$ of the total number of areas are shed more than fourteen times whilst $75 \%$ are shed only once. These show that there is a significant difference between the number of times each area is shed, which may well be interpreted as unfair. The causes of this result lie in the differences in order of magnitude of all areas' demand as well as the cost of load shedding associated to each area. Areas which are predominantly populated by industrial and commercial sectors were assigned with higher electricity demand than the other areas, and the cost of load shedding associated to these areas are also high. Thus these areas are expected to be shed less often than the others, so that the generated schedule exhibits low cost. 


$$
\lambda=1, \alpha=\beta=5
$$

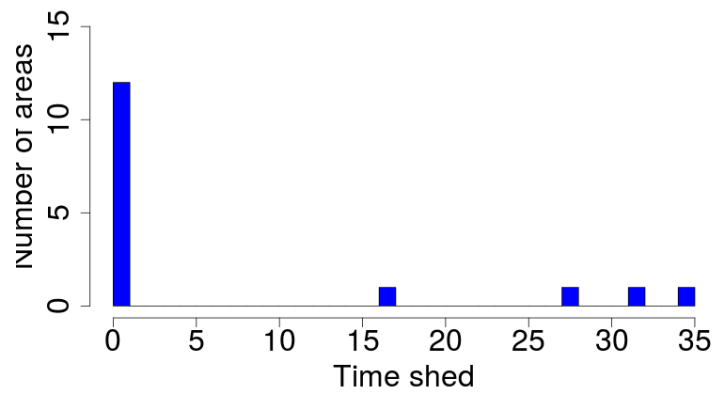

$$
\lambda=3, \alpha=\beta=5
$$

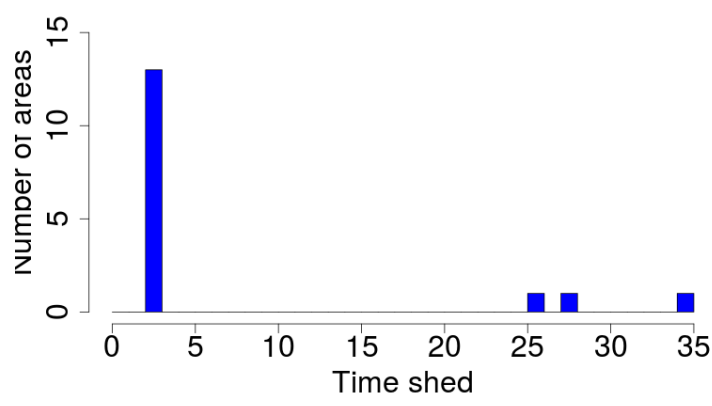

$$
\lambda=5, \alpha=\beta=5
$$

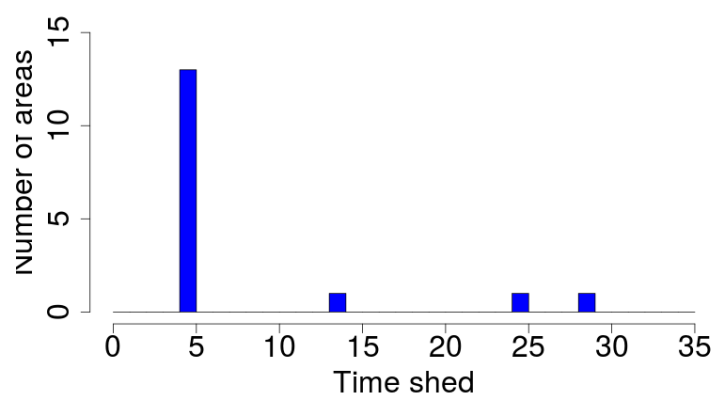

$$
\lambda=1, \alpha=\beta=3
$$

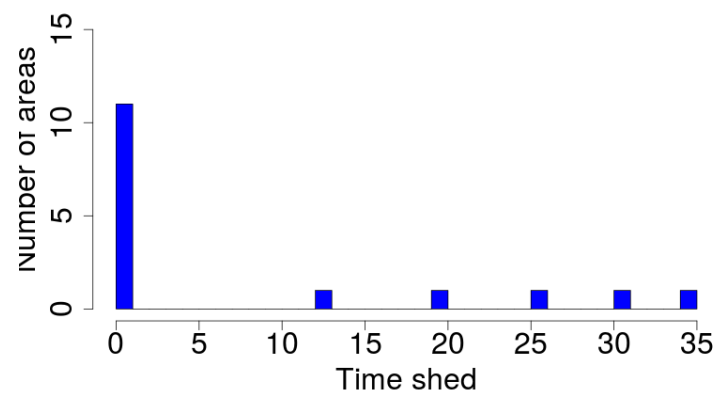

$$
\lambda=3, \alpha=\beta=3
$$

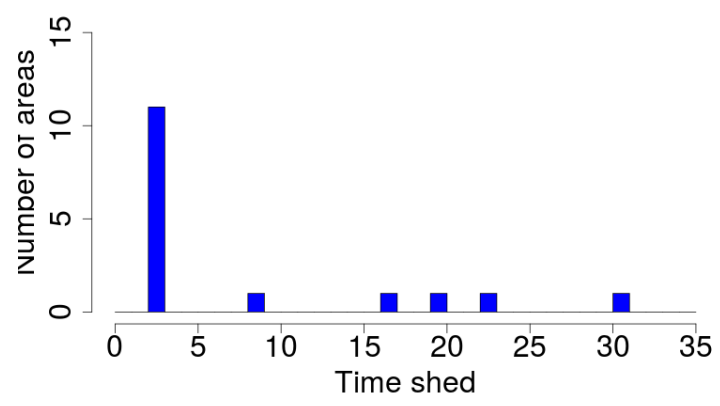

$$
\lambda=5, \alpha=\beta=3
$$

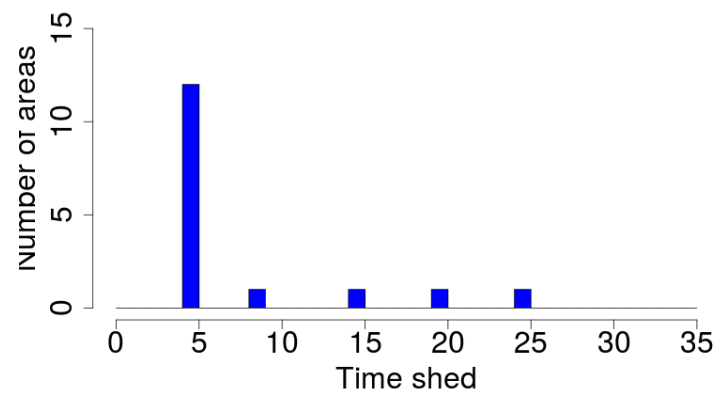

Figure 3: Distributions of the total number of times shed of all areas when solving the single objective model for different values of the parameters $\lambda, \alpha$ and $\beta$. 
A fair schedule would only be achieved with some sacrifices with respect to the total cost returned by the generated schedule or in the case where all areas are assigned with identical demand. In the later case, if it is assumed that all areas consume the same amount of electricity at any time period and that this electricity demand is equal to the required amount of load to be shed during that time period, then a fair schedule may be obtained as shown in Figure 4. For $\lambda=5, \alpha=\beta=1,87 \%$ of the total number of areas are shed 5 times, while the remaining areas are shed 7 times, in the returned schedule.

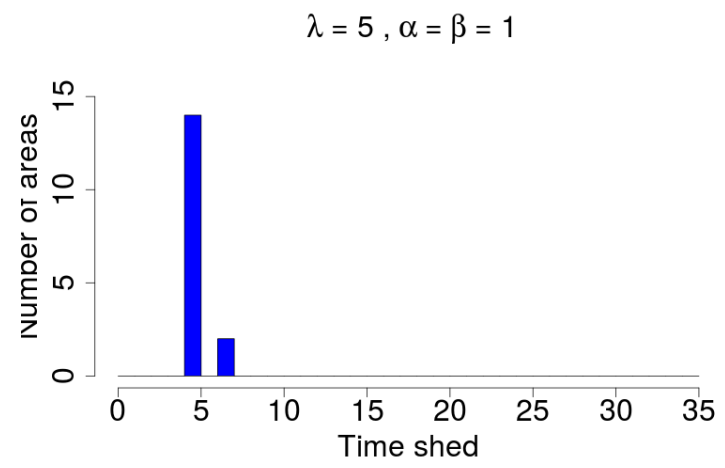

Figure 4: Distributions of the total number of times shed of all areas when solving the single objective model for $\lambda=5, \alpha=1$, and $\beta=1$, and $d_{i j k}=l_{j k}$ for $i \in\{1, \ldots, 16\}, j \in\{1, \ldots, 12\}$, and $k \in\{1, \ldots, 7\}$.

\subsection{Results obtained by the multiple objective model}

The sensitivity analysis of the results returned by the goal programming model with respect to changes in the preference parameters used as inputs to the model are shown in Figure 5. Results obtained from the first case study, in which the weight associated to the economic cost goal is varied, with an increasing magnitude from 1 to 7 , while the other weights are kept constant, are given in Figures 5(a), (c), and (e).

Increasing the weight associated with the economic cost goal leads to relatively small, but significant, decrease in the overall cost of the solution (see Figure 5(a)), while it results in a limited increase in the total deviation of the hourly rotational allocation goal from its target (see Figure 5(e)). This demonstrates that adding more weight on the cost goal enhances its importance, which results in a solution that achieves a low economic cost while sacrificing fairness allocation. The average number of times shed of all areas is, however, not significantly sensitive to the changes in the weight associated with the economic cost in this case, as shown in Figure 5(d). The majority of the areas are on average shed equally frequently.

Different results are obtained for the second case where the weights $\omega_{i k}^{\text {(time) }}(i \in\{1, \ldots, 16\}, k \in$ $\{1, \ldots, 7\})$ and $\omega_{i j}^{(\text {day })}(i \in\{1, \ldots, 16\}, j \in\{1, \ldots, 12\})$ associated to the daily and hourly rotational allocations goals were varied while keeping the remaining weights constant, as shown in Figures 5(b), (d), and (f). A 64-fold increase in the weight associated to the daily rotational allocation goal contributes to a $15 \%$ increase in the overall cost of the 


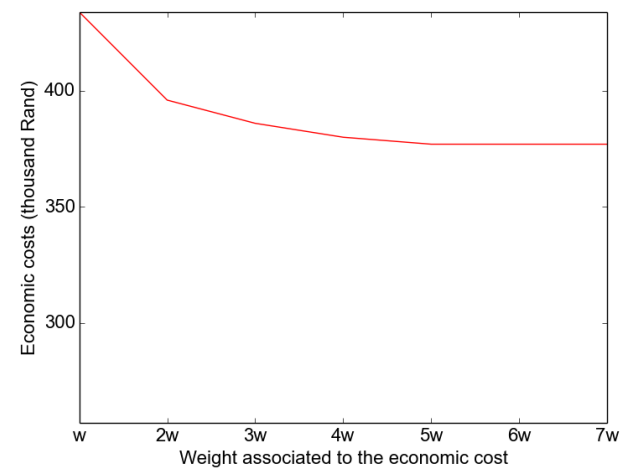

(a) Effect of varying the weight $\omega$ on the economic cost goal

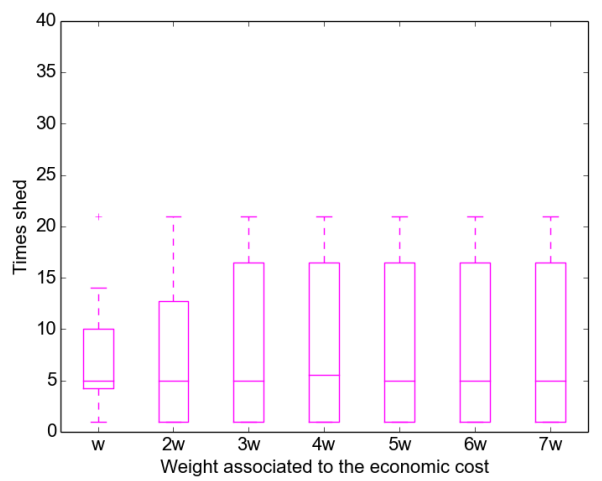

(c) Effect of varying the weight $\omega$ on the total number of times shed

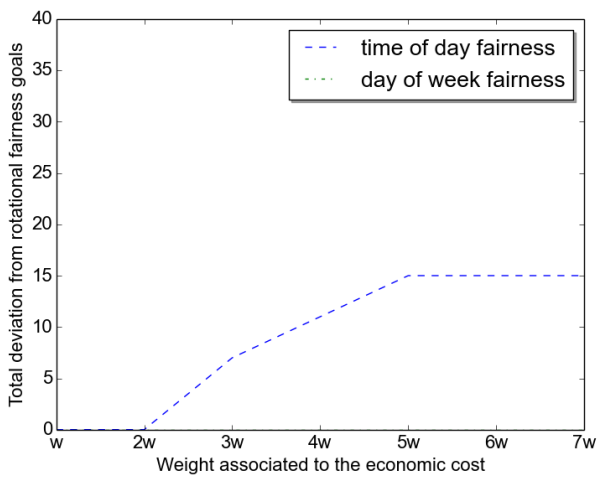

(e) Effect of varying the weight $\omega$ on the fairness allocations goals

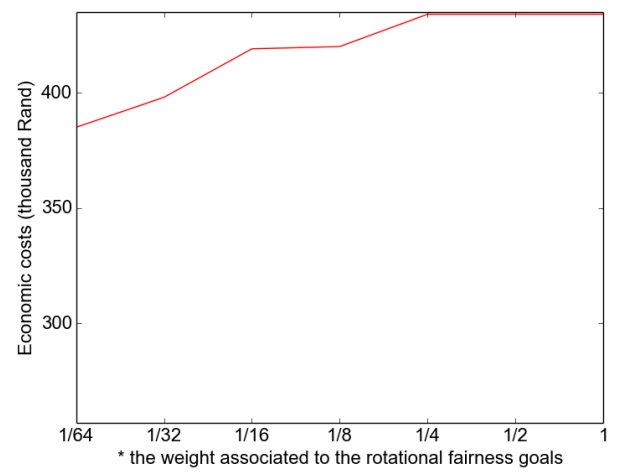

(b) Effect of varying $\omega_{i k}^{\text {(time) }}$ and $\omega_{i j}^{\text {(day) }}$ on the economic cost goal

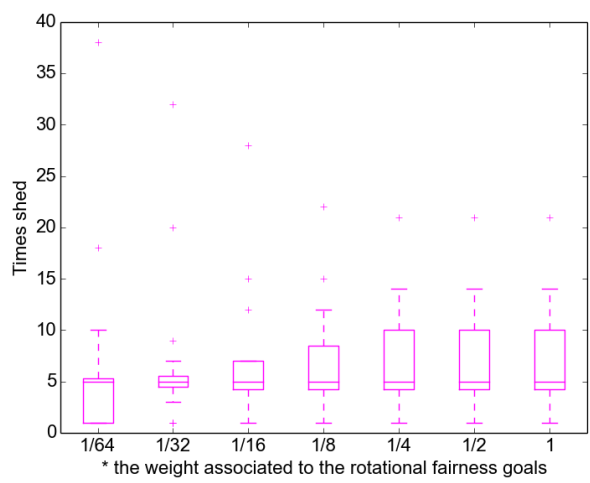

(d) Effect of varying $\omega_{i k}^{\text {(time) }}$ and $\omega_{i j}^{\text {(day) }}$ on the total number of times shed

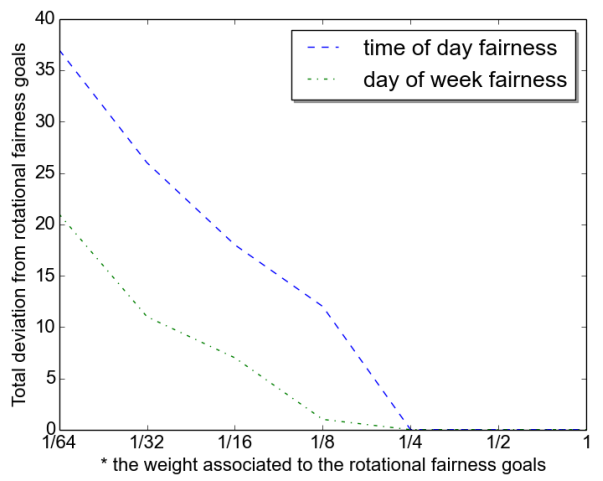

(f) Effect of varying $\omega_{i k}^{\text {(time) }}$ and $\omega_{i j}^{\text {(day) }}$ on the fairness allocations goals

Figure 5: The effect of varying the values of the various weights associated to all goals on a solution obtained by solving the multiple objective model. 
obtained solution (see Figure 5(b)). A significant drop in the total deviation of both the daily and hourly rotational allocation goals from their targets are also noticed in Figure $5(\mathrm{f})$. These results are expected as the rotational fairness goals are emphasized in this case. Moreover, the schedule is notably fair, the majority of all areas are on average shed equally frequently (see Figure 5(d)).

All these results show a clear trade-off between the various goals and objective functions. Although the trade-off between fairness and economic costs is a fairly obvious point, it is also important because it highlights the trade-off that decision makers must necessarily confront when creating a schedule. The current schedule for the City of Cape Town, for example, is likely to be relatively expensive because it is maximally fair [7].

\section{Conclusion}

Load shedding is inevitable when there is shortage in the power production levels and insufficient reserve margins to satisfy demand. The problem of how to generate feasible schedule that fairly allocates load shedding capacities over geographic areas was considered in this paper. Two programming models were proposed. The first model was a linear programming approach involving a single objective function, while the second model consisted of a goal programming approach. The objective was to emphasize the trade-off between fairness allocations and economic costs.

The two models were solved and evaluated in the context of realistic, but hypothetical, data. A limited experimental study based on sensitivity analysis with respect to the underlying parameters of the models was also conducted in order to assess their performances. Results indicated that a fair schedule comes at the cost of negative economic impacts, whereas low economic cost can only be achieved with some sacrifices to the fairness of the schedule. Decision makers would need to choose the schedule that best fits with their preferences.

Further follow-up research include implementation and assessment of the two models on real case studies. Moreover, all parameters involved in the models were assigned deterministic values in this paper. Stochastic versions of each of the models might thus be developed. Finally, these models generate schedules which only take into account future fairness. Past history, concerning the allocations of areas in the past schedule, was not considered. Possible future research is, therefore, to develop stochastic models which are able to generate schedules that are retrospectively fair, which would look back at the past distribution of load shedding (the number of times each area was shed) then correct any imbalances over some user-specified future time interval. The fact that some areas can offer other areas money to keep their electricity on more often and the other's electricity off more often can also be included in the model for further study.

\section{Acknowledgments}

This work was financially supported by the Deutscher Akademischer Austauschdienst German Academic Exchange Service (DAAD) together with the African Institute for Mathematical Sciences (AIMS) in the form of a bursary for the first author. 


\section{References}

[1] Adrian F, 2011, City of Cape Town, metropolitan municipality 199 from census 2011, [Online], [Cited November 21 $\left.{ }^{\text {st }}, 2018\right]$, Available from https://census2011.adrianfrith.com/place/199.

[2] Applegate D \& Cook W, 1991, A computational study of the job-shop scheduling problem, ORSA Journal on Computing, 3(2), pp. 149-156.

[3] BDO Consulting G, 1999, Energy intensive users group of southern africa, [Online], [Cited March $\left.7^{\text {th }}, 2017\right]$, Available from http://eiug.org.za/.

[4] Carpentier P, Gohen G, Culioli J \& Renaud A, 1996, Stochastic optimization of unit commitment: A new decomposition framework, IEEE Transactions on Power Systems, 11(2), pp. 1067-1073.

[5] Citizen T, 2019, Load shedding in 2007/08 cost economy estimated r50bn, [Online], [Cited March $\left.7^{\text {th }}, 2019\right]$, Available from https://citizen.co.za/news/south-africa/state-capture/2087259/ load-shedding-in-2007-08-cost-economy-estimated-r50bn/.

[6] Dahal KP, 2004, A review of maintenance scheduling approaches in deregulated power systems, Proceedings of the International Conference in Power Systems (ICPS), Kathmandu, pp. 565-570.

[7] City of Cape Town, Load shedding, 2018, [Online], [Cited November 21 ${ }^{\text {st }}, 2018$ ], Available from https://www . capetown.gov.za/en/electricity/Pages/LoadShedding . aspx.

[8] Froger A, Gendreau M, Mendoza Je, Pinson É \& Rousseau LM, 2016, Maintenance scheduling in the electricity industry: A literature review, European Journal of Operational Research, 251(3), pp. 695-706.

[9] Gupta JN, 1988, Two-stage, hybrid flowshop scheduling problem, Journal of the Operational Research Society, 39(4), pp. 359-364.

[10] MÉndez CA \& CERdÁ J, 2003, Dynamic scheduling in multiproduct batch plants, Computers \& Chemical Engineering, 27(8), pp. 1247-1259.

[11] Parvania M, Fotuhi-Firuzabad M, Aminifar F \& Abiri-Jahromi A, 2010, Reliabilityconstrained unit commitment using stochastic mixed-integer programming, $11^{\text {th }}$ International Conference on Probabilistic Methods Applied to Power Systems (PMAPS), pp. 200-205.

[12] Pretorius I, Piketh S \& Burger R, 2015, The impact of the South African energy crisis on emissions, WIT Transactions on Ecology and Environment, 198, pp. 255-264.

[13] Ruiz PA, Philbrick CR, Zak E, Cheung KW \& Sauer PW, 2009, Uncertainty management in the unit commitment problem, IEEE Transactions on Power Systems, 24(2), pp. 642-651.

[14] Trilling L, Guinet A \& Le Magny D, 2006, Nurse scheduling using integer linear programming and constraint programming, IFAC Proceedings Volumes, 39(3), pp. 671-676.

[15] Wang X \& James M, 1994, Modern power system planning, McGraw-Hill, London.

[16] Wood AJ \& Wollenberg BF, 2012, Power generation, operation, and control, John Wiley \& Sons.

[17] Xiong P \& Jirutitijaroen P, 2013, A stochastic optimization formulation of unit commitment with reliability constraints, IEEE Transactions on Smart Grid, 4(4), pp. 2200-2208. 